\title{
UM SISTEMA PARA APOIO \\ À FORMAÇÃO DE EMPRESAS \\ VIRTUAIS BASEADA EM RECURSOS DE CHÃO-DE-FÁBRICA
}

\section{GESTÃO \\ $\&$ \\ PRODUÇÃO}

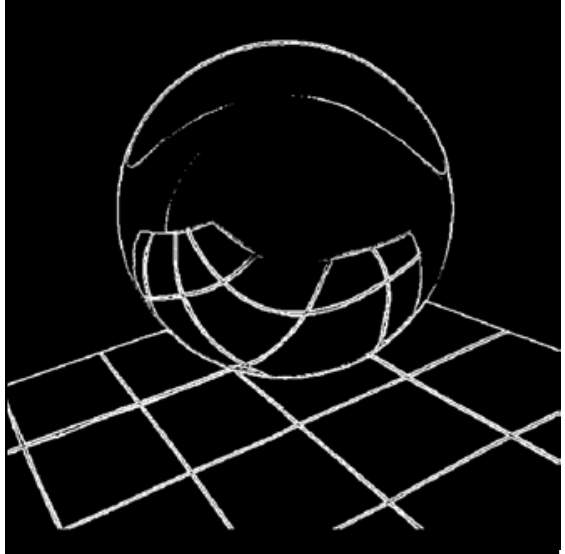

v.6, n.2, p. 79-86, ago. 1999

Carlos Frederico Bremer

Engenharia de Produção Mecânica Escola de Engenharia de São Carlos - USP Av. Dr. Carlos Botelho,1465 - São Carlos - SP Tel.: (016) 273-9425

E-mail: cfbremer@prod.eesc.sc.usp.br

\section{Resumo}

A agilidade para explorar novas oportunidades de negócios é um fator chave para que as empresas mantenham sua competitividade. Os avanços das tecnologias de informação, tais como a Internet, possibilita o trabalho conjunto de empresas, mesmo estando separadas geograficamente. Essas tecnologias possibilitam novas formas de cooperação, como por exemplo Empresas Virtuais. Esse artigo visa apresentar o sistema VISHOF - Virtual Shop Floor - que possibilita que as empresas compartilhem seus recursos de chão-de-fábrica, no intuito de criar Empresas Virtuais. Esse sistema é um software cujo acesso e operação são realizado pela Internet, através de um browser.

\section{Palavras-chave: empresas virtuais, Internet, competências essenciais.}

\section{Introdução}

Dara manterem-se competitivas, as empresas Têm modificado a maneira de gerir seus negócios, passando a valorizar mais as necessidades de seus mercados consumidores, e direcionando esforços para o foco principal de seu negócio, ou seja, sua core competence (competência essencial).
Como resultado disso, as empresas passaram a terceirizar atividades que não agregam valor, trouxeram o cliente para dentro da empresa, passaram a investir em gestão da qualidade, adotaram e passaram a enxergar a empresa numa visão de processos de negócios. Todas essas ações trouxeram competitividade para as empresas, mas já se tornam uma prática comum 
entre elas. Outro modo de ser competitiva é tornar-se ágil o suficiente para conseguir explorar oportunidades de negócio.

$\mathrm{O}$ avanço das tecnologias de informação e comunicação possibilitou uma redução significativa nos custos de transação, criando assim um ambiente propício para ocorrência de cooperações entre empresas. Essas cooperações ocorrem dentro de um ambiente chamado organizações virtuais e são denominadas empresas virtuais.

As empresas virtuais podem ser definidas como uma rede temporária de empresas unidas pela tecnologia de informação para explorarem conjuntamente um mercado e dividirem riscos e custos, além de somarem competências (BYRNE，1993). GORANSON (1995) define empresas virtuais como uma reunião oportunista de empresas que visam um determinado objetivo comum.

Não se limitando apenas às definições de empresas virtuais, é possível identificar quatro fatores que caracterizam uma empresa virtual:

1- Temporariedade: a empresa virtual opera dentro do período de tempo que durar a oportunidade de negócio (GOLDMAN, 1995; GORANSON, 1995), porém, caso esta oportunidade de negócio seja duradoura, pode-se estender a cooperação para outras formas mais sólidas, como uma joint venture, por exemplo.

2- Confiança: as ações de cooperação entre as empresas virtuais são baseadas na relação de confiança existente entre as empresas cooperadoras (EVERSHEIM, 1996; FUKUYAMA, 1997), o que gera um ganho de tempo nas ações conjuntas, pois elimina-se tramites burocráticos.

3- Tecnologia de Informação: as empresas virtuais utilizam de forma intensiva os recursos da tecnologia de informação e comunicação (HERBST, 1996; PICOT, 1996), reduzindo-se, assim, os custos de transação e aumentando o espaço economicamente viável de uma cooperação.

4- Core Competence (Competência Essencial): segundo o projeto MOTION (TERHAAG,
1996), uma das definições de competência essencial é o conjunto de tarefas (habilidades) que garantem o sucesso e a continuidade da existência de uma empresa. E entre as empresas virtuais, objetiva-se o uso das competências essenciais de cada um dos parceiros.

Essas quatro características podem ser observadas na Figura 1.

O objetivo do presente artigo é apresentar o sistema VISHOF - Virtual Shop Floor - um sistema que possibilita o compartilhamento de recursos de chão-de-fábrica entre os participantes de uma empresa virtual. Esse sistema é composto de um software desenvolvido para o ambiente Internet. Para o acesso e operação pode-se utilizar um Intranet, limitando assim o acesso de empresas que não façam parte da Organização Virtual. Além disso, é necessário apenas um paginador Web (browser) para sua operação.

\section{Virtual Shop Floor - VISHOF}

$\mathrm{O}$ sistema VISHOF foi baseado na tecnologia Internet cujo objetivo principal é gerenciar dados sobre as competências de chão-de-fábrica de empresas de uma determinada região. Convencionou-se chamar competências de chãode-fábrica os recursos utilizados para realização de operações num chão-de-fábrica, tais como máquinas operatrizes, instrumentos de medição, dispositivos, etc.

\subsection{Funcionamento do Sistema}

O sistema disponibiliza ao usuário três tipos de serviço: busca de recurso, administração de informações e informações estatísticas.

A busca de recurso pode ser realizada de duas maneiras: uma por meio de uma consulta estruturada na base de dados de informações do sistema, e a outra, informando-se o nome exato do recurso. A consulta estruturada inicia-se com a escolha do grupo de recurso, como por exemplo: máquinas ferramentas, instrumentos de medição, etc. Uma vez selecionado o grupo do recurso, 


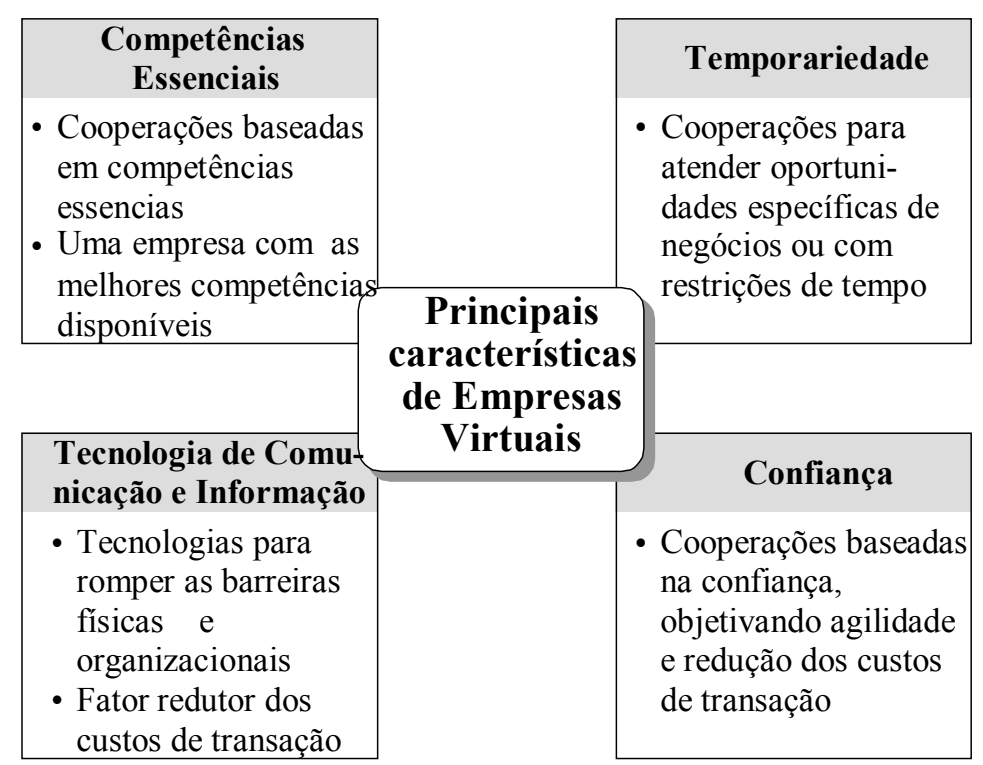

Figura 1 - Características principais das empresas virtuais.

pode-se então especificar a classe do recurso. Exemplos de classes para o grupo máquinas ferramentas são: tornos, fresas, furadeiras, mandriladoras, entre outros. Dentro de cada classe ainda existem os tipos de recurso, como por exemplo, para um torno temos: vertical, horizontal, revólver, com comando numérico. Cabe salientar que podemos ter um torno horizontal, revólver com comando numérico. A especificação do tipo do recurso tem o objetivo de reunir as principais características para que o usuário possa encontrar o recurso desejado.

Depois de selecionar o tipo do recurso, o sistema irá apresentar uma série de características, tais como: potência, distância entre pontos, barramento, velocidades, entre outras. O usuário poderá então fornecer as características detalhadas dos recurso necessário. Para sistematização das características de cada tipo de máquina utilizou-se uma carta de especificação de máquinas no padrão AWF (Auschuss für Wirtschaftliche Fertigung).

Outra maneira de efetuar-se a busca de um recurso é por meio de suas informações organizacionais, ou seja, fabricante, modelo, nome do recurso. Uma vez inseridas estas informações, o sistema realiza uma busca em sua base de dados e traz o número de recursos que respondem às especificações que foram inseridas anteriormente. Caso o número de recursos com essas características seja alto, é possível refinar a busca, chegando a uma quantidade menor de recursos. Pode-se, então, consultar esses recursos e levantar qual a capacidade disponível, dentro de qual período essa capacidade está disponível, qual a empresa proprietária do recurso e quem é o contato dentro da empresa. A Figura 2 mostra uma tela do sistema VISHOF.

O serviço de administração de informações permite que a empresa usuária, que possua recursos cadastrados no sistema VISHOF, possa fazer a manutenção dessas informações. Por este serviço, a empresa poderá alterar a capacidade disponível de seus recursos, retirar determinado recurso da base de dados ou inserir um novo recurso. Cada empresa usuária designa usuários para operarem o sistema e cada usuário possui permissões de acesso. Quando o usuário entra no sistema, esse identifica a qual empresa o usuário pertence, quais os recursos que essas empresa possui dentro do sistema, e qual as autorizações do usuário. Desse modo é possível que a empresa rapidamente encontre seus recursos para atualização das informações. 


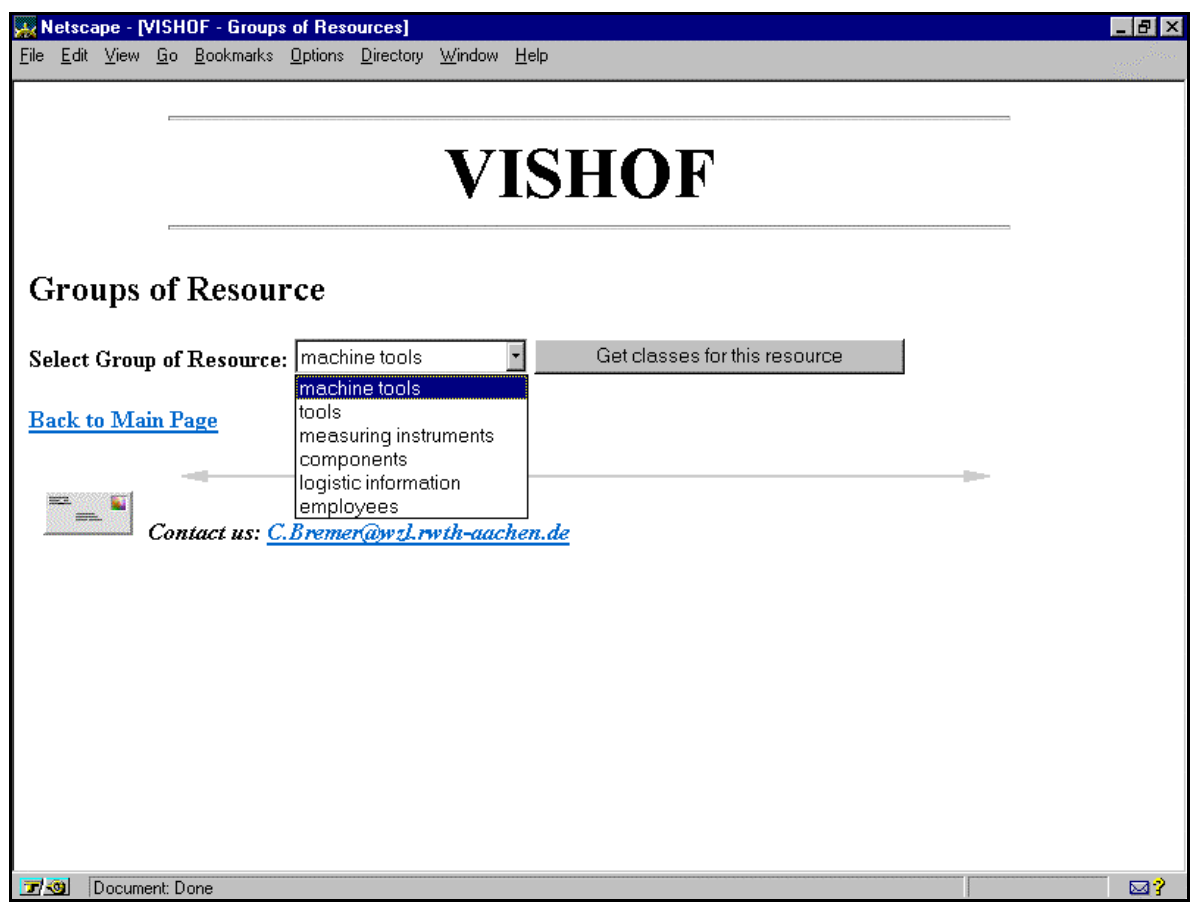

Figura 2 - Tela do sistema VISHOF.

As regras para utilização do sistema serão apresentadas em detalhes na seção seguinte.

O serviço de informações estatísticas fornece diversas estatísticas ajudando tanto as empresas como o planejamento industrial de uma região. Entre as informações obtidas pelo sistema, é possível saber quais os recursos mais procurados e quais são os recursos mais comuns na região. Uma empresa pode levantar a necessidade de aquisição de uma máquina por meio de suas estatísticas de procura do recurso. Para o desenvolvimento de uma região é possível que sejam mapeados quais os recursos que possuem uma alta demanda, e baixa quantidade.

\subsection{Utilização do Sistema}

Para utilização do sistema, convencionou-se que:

- existem empresas numa determinada região geográfica que possuem recursos, e a utilização desses recursos não é constante ao longo do tempo, podendo então ocorrer ociosidade de um recurso, ou falta do mesmo;
- existe um corretor de informações (Information Broker), podendo este ser uma organização privada ou não, cuja função é levantar, administrar e disseminar informações sobre os recursos de chão-de-fábrica de uma certa região. Estas informações sobre os recursos de chão-de-fábrica seriam disseminadas entre empresas que se cadastrariam nesta organização, e tornaram-se usuárias do sistema VISHOF e;

- o(s) responsável(eis) pela administração dos recursos de chão-de-fábrica têm liberdade de tomar decisões a respeito de venda ou compra de capacidade de e para terceiros.

Das convenções fica caracterizado a existência do corretor de informações, o Information Broker, que é a entidade que administra o sistema VISHOF, e a existência das empresas usuárias do sistema. A relação entres esses elementos é apresentada na Figura 3.

Cabem ao Information Broker as seguintes atividades:

- garantir o funcionamento do sistema pela manutenção adequada dos sistemas de banco 


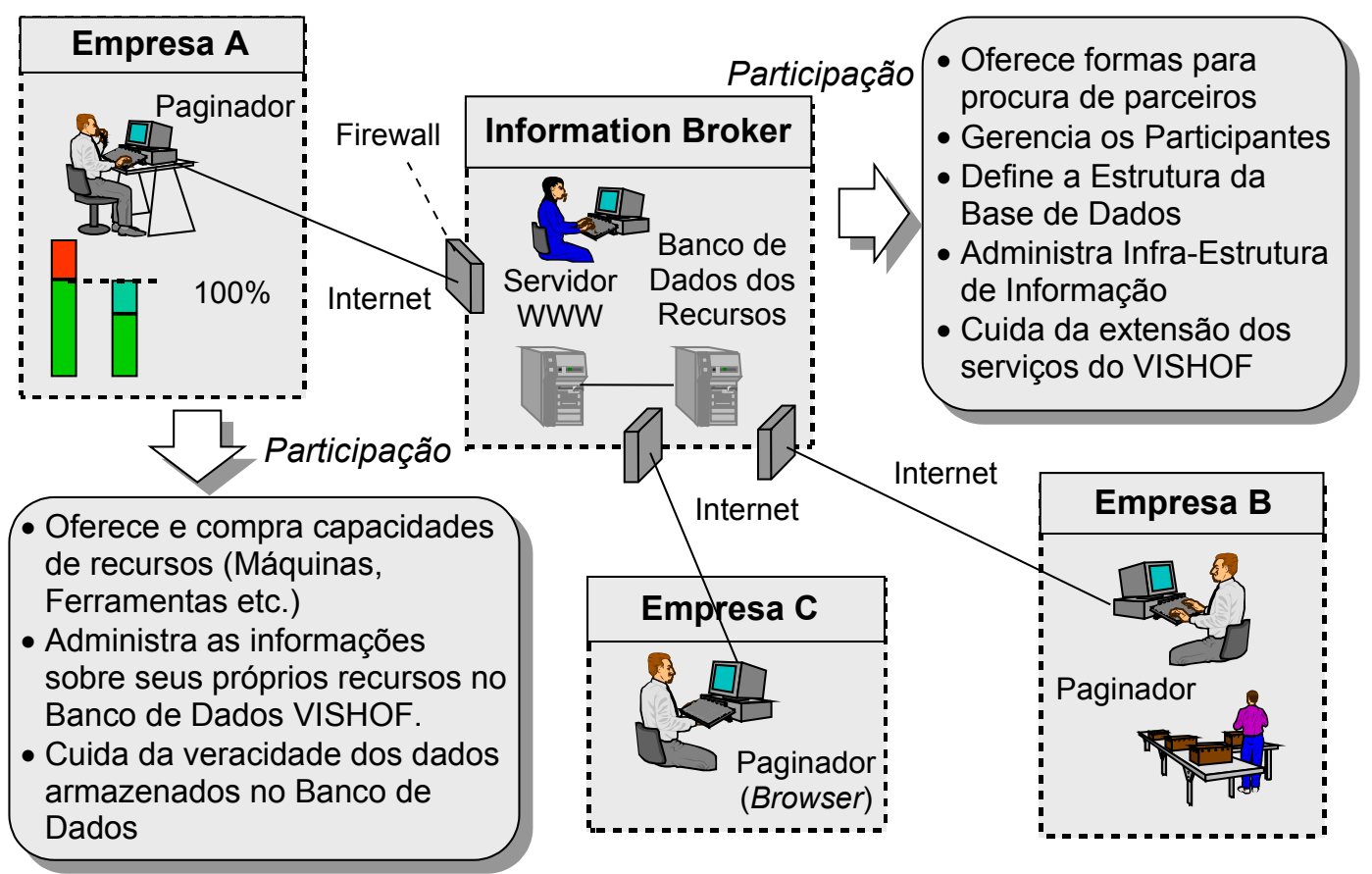

Figura 3 - Conceito do sistema VISHOF.

de dados, bem como do servidor World Wide $W e b$;

- levantar as competências de chão-de-fábrica das empresas e realizar o primeiro cadastramento dos recursos de cada empresa no sistema VISHOF;

- garantir que as permissões de acessos funcionem corretamente, ou seja, fazer com que uma empresa tenha acesso para manutenção somente de seus recursos.

A empresa usuária é responsável pelas seguinte atividades:

- garantir a veracidade e manutenção das informações que constam na base de dados do sistema;

- atualizar dentro do sistema as informações referentes à capacidade disponível para venda de cada recursos, toda vez que houver uma mudança na disponibilidade de capacidade dentro da empresa.

Para uma empresa tornar-se usuária do sistema VISHOF é necessário que seja cadastrada pelo Information Broker. Esse cadastro consiste do levantamento e introdução na base de dados dos recursos que a empresa se dispõe a compartilhar. Além disso, é necessário que a empresa tenha um acesso a Internet e um paginador web (browser) para operar o sistema.

\section{Arquitetura do Sistema VISHOF}

耳 ssa seção descreve as características - técnicas utilizadas no desenvolvimento do sistema VISHOF.

A arquitetura utilizada para o desenvolvimento desse sistema visa atender às necessidades de operação descritas na seção anterior. Para isso, o sistema consiste de uma Intranet, na qual cada empresa usuária pode buscar informações sobre recursos que esteja necessitando.

O fluxo de informações dentro do sistema está estruturado do seguinte modo: o usuário do sistema envia uma chamada (através do browser) num padrão HTTP (Hypertext Transfer Protocol). Essa chamada chega então ao servidor de páginas Web, e através de processo CGI-bin (Common Gateway Interface) é realizado então uma busca via ODBC (Open Data Base 


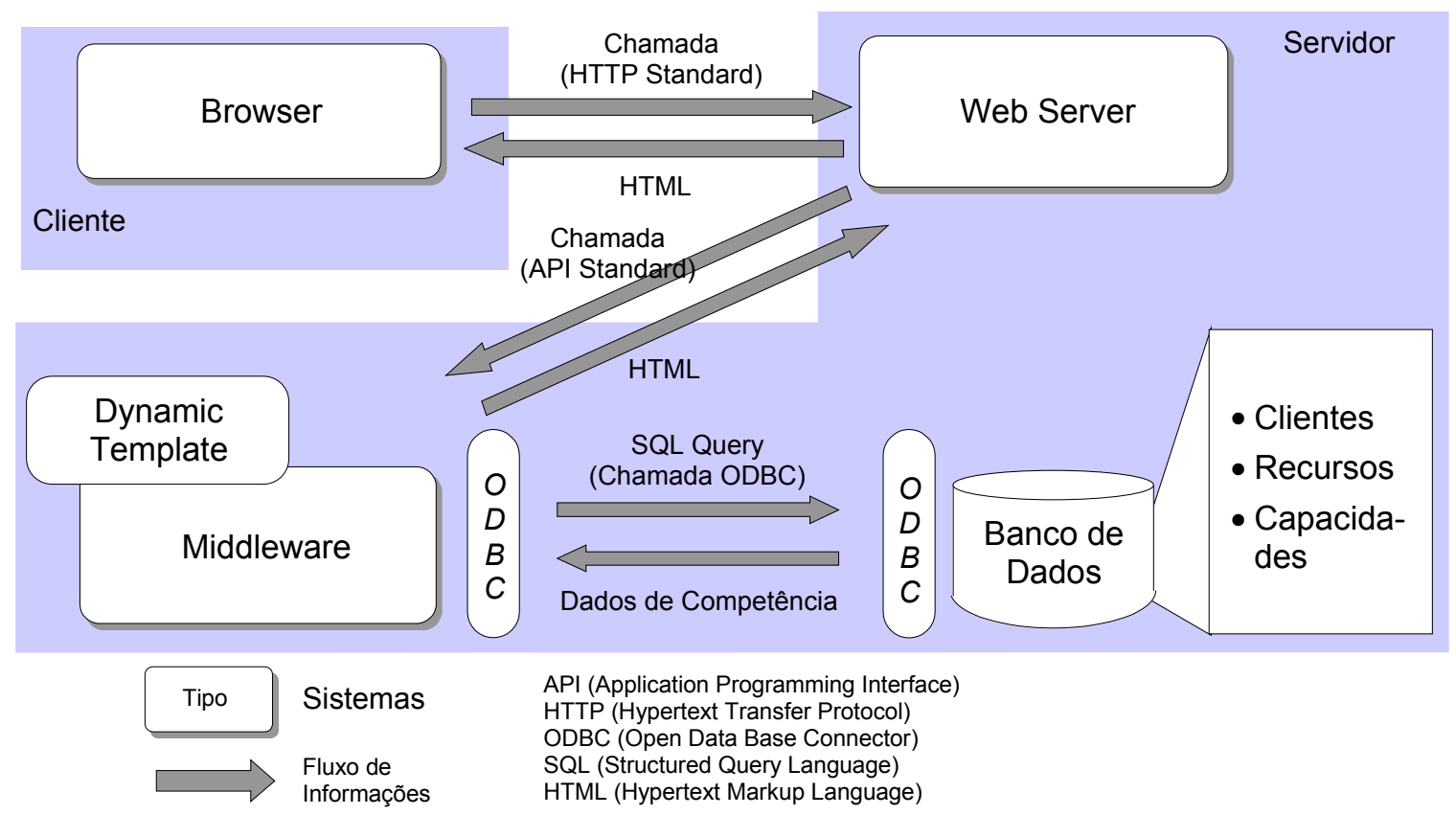

Figura 4 - Arquitetura do sistema VISHOF.

Connector) dentro da base de dados, utilizandose o padrão SQL (Structured Query Language). Esse processo CGI é realizado por um middleware que consiste de uma ferramenta de programação. O resultado da consulta ao banco de dados é enviado de volta ao middleware, onde são geradas páginas Web contendo as informações requisitadas. As páginas estão num formato HTML (Hypertext Markup Language) e são geradas dinamicamente pelo middleware. $\mathrm{O}$ fluxo de informações pode ser observado na Figura 4.

O sistema foi desenvolvido para ser utilizado na Internet como sendo uma Intranet. Para garantir a segurança do sistema num primeiro nível, pode-se restringir, pelo servidor Web, o acesso pelo endereço IP (Internet Protocol), necessitando para isso um usuário e uma senha, e ainda nesse nível pode-se restringir as permissões de acesso de cada usuário através do sistema operacional. Além disso, o servidor Web pode utilizar o protocolo de segurança Secure Socket Layer (SSL), que realiza o processo de criptografia de informações entre servidores.
Esse protocolo é um padrão industrial que utiliza tecnologia de criptografia para transmissão de dados entre servidores, assegurando uma via segura na transferência de dados.

\section{Aspectos Favoráveis}

$\mathrm{O}$ sistema VISHOF apresenta vários aspectos favoráveis tanto para uma empresa usuária quanto para uma determinada região. Com relação à operação do sistema, o VISHOF apresenta a vantagem de poder ser operado via Internet, fazendo com que o usuário necessite apenas de um browser. Essa característica é importante pois garante benefícios, tais como baixo custo na implementação do sistema, visto que o usuário precisaria somente de um acesso à Internet, e de um browser que pode ser obtido gratuitamente através da rede. Outro aspecto favorável é a interface amigável proporcionada pelo sistema, visto que a operação do sistema é basicamente a operação de um web site.

Entre os aspectos favoráveis para uma empresa usuária do sistema, tem-se um aumento no 
nível de utilização dos recursos internos da empresa, possibilitado pela venda de capacidade de produção ociosa. Outro fator importante é a possibilidade de se aumentar a competitividade da empresa, uma vez que ela tem acesso a outras competências de chão-de-fábrica que possibilitam a busca e atuação em oportunidades de negócio. Além desses fatores podemos ainda citar:

- possibilidade de se encontrar competências específicas: o sistema de informação VISHOF pode ser um meio rápido de se encontrar recursos específicos de produção na rede de parceiros. Tais recursos podem ser máquinas específicas, equipamentos de medição ou até processos de fabricação; e

- constituição de uma base para cooperações: o VISHOF pode estimular as empresas participantes a formarem cooperações como forma de melhoria da competitividade.

As vantagens para uma região podem ser resumidas nos seguintes fatores:

- aumento da sinergia entre as empresas da região: cria-se uma identidade e contribui-se para o aumento da capacidade produtiva de toda a região; $\mathrm{e}$

- estatísticas sobre os principais recursos subtilizados ou escassos: pelas estatísticas de utilização do sistema de busca de recursos, o corretor de informações pode saber quais os recursos mais escassos ou subtilizados em uma região. Estas estatísticas podem ser úteis para instituições regionais, institutos de pesquisas ou mesmo para a detecção de nichos de mercados de bens de produção e serviços não atendidos.

Com o aumento da sinergia entre as empresas de um determinada região, cria-se uma relação de confiança entre as empresa, gerando um ambiente favorável à ocorrência de cooperações do tipo empresas virtuais. Dessa maneira, obtém-se um aumento de competitividade da região como um todo.

\section{Conclusões}

$\mathrm{O}$ sistema VISHOF mostra-se uma importante ferramenta para cooperação baseado nos recursos de chão-de-fábrica, e conseqüentemente para formação de empresas virtuais. O sistema VISHOF será implantado na VIRTEC. A VIRTEC é a primeira organização virtual desenvolvida no Brasil. É constituída de nove empresas da região de São Carlos, cuja coordenação é feita pelo grupo de empresas virtuais do Núcleo de Manufatura Avançada da USP - São Carlos.

Atualmente estuda-se a expansão do sistema. O projeto de expansão do sistema VISHOF consiste da incorporação das demais competências encontradas dentro de uma organização. Essas competências foram definidas como: produtos, processos e recursos. Portanto, novas funcionalidades estarão sendo incorporadas ao sistema VISHOF de modo que será possível a busca de informações referentes a produtos e processo de negócios de empresas de uma determinada região.

\section{Referências Bibliográficas}

BYRNE, J.A.: "Virtual Corporation", Business Week, February 8, 1993.

EVERSHEIM, W.; BREMER, C. \& KAMPMEYER, J.: "Requirements for Virtual Enterprise Management In Developing Countries", International Conference on Engineering and Technology Management, Vancouver, Canada, p.84-88, 1996.

FUKUYAMA, F.: “Confiança ainda é fundamental”, Revista Exame, p.72, Março, 1997.
GOLDMAN, S.; NAGEL, R. \& PREISS, K.: Agile Competitors - Concorrência, Organizações Virtuais e Estratégias para valorizar o Cliente, Editora Érica, 1995, 374p.

GORANSON, H.T.: “Agile Virtual Enterprise: Best Agile Practice Reference Base", http://snap.org, 1995.

HERBST, D.: "Kommunikation wird zum Schlüsselfaktor”. PC Magazin, 25, p.36-37, 1996. 
PICOT, A.; REICHWALD, R. \& WIGAND R.: "Die Grenzenlose Unternehmung", Gabler Verlag, 1996, 579p.
TERHAAG, O.; DRESSE, S. \& KÖLSCHEID, W.:

"Model for transforming, identifying and optimizing core processes (MOTION)", Final Report for Work Package 1.2, August, 1996.

\title{
A SYSTEM BASED ON SHOP FLOOR RESOURCES TO SUPPORT THE FORMATION OF VIRTUAL ENTERPRISES
}

\begin{abstract}
The agility to exploit new business opportunities is key to success for an enterprise to keep its competitiveness. The information technology advances, such as the Internet, allow enterprises to work together effectively even if they are geographically separated. These technologies facilitate new forms of cooperation, like Virtual Enterprises. This paper describes the VISHOF, Virtual Shop Floor system, that enables enterprises to share their shop floor resources to form Virtual Enterprises. This system is a piece of software for which access and operation is made via the Internet with a browser.
\end{abstract}

Key words: virtual enterprise, Internet, core competence. 